\title{
Cholesterol gallstone disease: focusing on the role of gallbladder
}

\author{
Yongsheng Chen, Jing Kong and Shuodong Wu
}

Gallstone disease (GSD) is one of the most common biliary tract diseases worldwide in which both genetic and environmental factors have roles in its pathogenesis. Biliary cholesterol supersaturation from metabolic defects in the liver is traditionally seen as the main pathogenic factor. Recently, there have been renewed investigative interests in the downstream events that occur in gallbladder lithogenesis. This article focuses on the role of the gallbladder in the pathogenesis of cholesterol GSD (CGD). Various conditions affecting the crystallization process are discussed, such as gallbladder motility, concentrating function, lipid transport, and an imbalance between pro-nucleating and nucleation inhibiting proteins.

Laboratory Investigation (2015) 95, 124-131; doi:10.1038/labinvest.2014.140; published online 15 December 2014

\section{INTRODUCTION}

The pathogenesis of cholesterol gallstone disease (CGD) has long been an enigma. During the first half of the last century, cholelithiasis was regarded to be due to gallbladder inflammatory disease, resulting in desquamation of cells and the production of abnormal substances. ${ }^{1}$ After Small and Rapo ${ }^{2}$ elucidated the physical-chemical basis of cholesterol crystallization, cholesterol gallstones were then considered to be the consequence of metabolic defects in the liver. Most efforts to understand this process have thus focused on mechanisms that govern the hepatic secretion of biliary cholesterol, phospholipids, and bile salts. Currently, hepatic secretion of cholesterol-supersaturated bile is regarded as a prerequisite for CGD. However, despite its obviously crucial role in lithogenesis, there has been limited research on the downstream events that occur in the gallbladder.

Until recently, renewed interest and considerable investigative efforts have been paid to the involvement of the gallbladder in CGD. ${ }^{3,4}$ Impaired gallbladder emptying, enhanced concentrating function, abnormal lipid transport, and mucus hypersecretion, all presumably secondary to the exposure of the gallbladder to lithogenic bile, have each been identified as additional etiologic factors in CGD. Although lithogenic bile secreted by the liver is now thought to be the main culprit in gallstone formation, the gallbladder is by no means an innocent bystander. As such, in this review we will focus on the role of the gallbladder in the formation of cholesterol gallstones.

\section{GALLBLADDER MOTILITY}

The gallbladder has motor function, with $20-30 \%$ emptying at 1- to $2-\mathrm{h}$ intervals during the fasting state and $70-80 \%$ emptying after stimulation by cholecystokinin (CCK) during a meal. The interplay between gallbladder contraction and relaxation has an important role in driving the flow of bile salts in enterohepatic circulation and facilitating the absorption of lipids and fat-soluble vitamins. However, the motor function of gallbladder smooth muscle (GBSM) becomes impaired during cholesterol gallstone formation in patients and animal models, which exhibit increased fasting gallbladder residual volume as well as reduced contractility in response to CCK. ${ }^{5,6}$ Among the many factors involved in the pathogenesis of CGD, gallbladder hypomotility and the resultant prolonged stasis of lithogenic bile seem to be the most significant. This dysfunction is a very early event in the development of CGD, which occurs within a few days after the onset of the lithogenic diet and gets worse as the disease progresses from microlithiasis to gallstones. ${ }^{7,8}$ Improving gallbladder motility with a prokinetic agent such as cisapride or erythromycin leads to a significant reduction in cholesterol crystallization in animals on a high cholesterol diet. ${ }^{9,10}$ On the other hand, administration of a CCK antagonist (MK-329) can lead to gallbladder stasis and enhanced gallstone formation in cholesterol-fed animals. ${ }^{11}$ Studies in patients after extracorporeal shock-wave lithotripsy have shown that more severely impaired gallbladder emptying was associated with a higher recurrence of gallstones. ${ }^{12}$ However,

Department of General Surgery, Shengjing Hospital of China Medical University, Shenyang, China

Correspondence: Dr S Wu, MD, PhD, Department of General Surgery, Shengjing Hospital of China Medical University, No. 36, San Hao Street, Shenyang 110004, China. E-mail: surgeon_cys@163.com

Received 1 September 2014; revised 11 October 2014; accepted 15 October 2014 
gallbladders with pigment stones do not develop defects in contraction like those seen with cholesterol gallstones. ${ }^{13}$ Taken together, these findings suggest that impaired gallbladder motility is an important contributing factor in CGD.

Impaired gallbladder emptying may result in prolonged stasis of lithogenic bile in the gallbladder, thus allowing more time and a permissive environment for cholesterol crystallization and aggregation of that cholesterol into macroscopic gallstones. A longer stay of bile in the gallbladder may lead to its hyperconcentration by gallbladder epithelium. Cholesterol is inclined to precipitate from gallbladder bile of a higher lipid concentration (see details in ion and water absorption). In addition, when a large proportion of the bile salt pool is entrapped within the sluggish gallbladder, the cholesterol saturation index (CSI) of the newly secreted hepatic bile is higher due to the relatively lower bile salt secretion and relatively higher cholesterol secretion. This phenomenon further promotes cholesterol precipitation. ${ }^{5}$

In the last two decades, investigators have attempted to address the question of what causes gallbladder hypomotility. Normal contraction of GBSM involves the binding of CCK to its receptors on the plasma membrane (PM) and subsequent activation of Gi-3 protein. ${ }^{14}$ A large body of evidence has shown reduced muscle contractility after stimulation by CCK in gallbladders with cholesterol gallstones. ${ }^{5,13}$ These findings are in agreement with a study that showed that gallbladders with cholesterol stones had significantly lower levels of ${ }^{125}$ I-labeled CCK- 8 binding to receptors, suggesting that there may be fewer CCK-1 receptors (CCK-1 R) available for binding on the PM. ${ }^{15}$ However, the defect in contraction can be corrected when PM receptors are bypassed by stimulation with $G$ protein activator or secondary messengers such as diacylglycerol. ${ }^{16}$ On closer inspection, these abnormalities are related to the presence of excessive cholesterol in the PM of GBSM and can be corrected by incubating muscle cells with cholesterol-free liposome carriers that remove the excess cholesterol. ${ }^{17}$ These findings point to receptor abnormalities in the PM of GBSM that may be caused by lithogenic bile. ${ }^{18}$

The mechanisms by which lithogenic bile might cause these alterations are not fully elucidated. However, there are a number of findings that provide some important insight. Lipid analysis showed that lithogenic bile contains increased levels of cholesterol and hydrophobic bile salts, both of which have inhibitory effects on GBSM contraction but via different mechanisms. Cholesterol supersaturation in bile may facilitate its diffusion across the gallbladder epithelium, where it is readily incorporated into the PM of GBSM. Cholesterol enrichment in the PM results in a marked reduction in spontaneous action potentials and voltage-activated $\mathrm{Ca}^{2+}$ currents in muscle cells. Therefore, basal tone and agonistinduced contractile properties of GBSM are attenuated. ${ }^{19}$ In addition, excess cholesterol can decrease the fluidity of PM, particularly in caveolar domains that participate in the internalization of most receptors following stimulation by their respective agonists. High caveolar cholesterol levels block caveolin-3 (CAV-3) phosphorylation required to deliver CAV-3 protein-CCK-1 $\mathrm{R}-\mathrm{G}_{\mathrm{i}-3}$ complexes to recycling endosomes. Their sequestration within caveolae further decreases the turnover of CCK-1 R from endosomes to PM; this leads to fewer CCK-1 $\mathrm{R}$ available for CCK-8 binding and weaker muscle contraction. ${ }^{20,21}$ Cholesterol overabundance in PM may also impair cytoprotective mechanisms of GBSM by decreasing the binding of PGE2 to its receptors in the same manner as CCK-1 R. As a result, the catalase response and degree of free radical inactivation is lower despite a similar PGE2 level. ${ }^{22,23}$ Hydrophobic bile salts inhibit GBSM contraction by generating free radicals $\left(\mathrm{H}_{2} \mathrm{O}_{2}\right)$ that damage PM receptors and then inhibit CCK-induced contraction. ${ }^{24}$ The demonstration that $G$ protein-coupled bile acid receptor (GPBAR1) is expressed in GBSM provides us a novel mechanism for gallbladder hypomotility. GPBAR1, also known as TGR5, is a G protein-coupled cell-surface receptor that is activated by both primary and secondary bile acids. This receptor is expressed in gallbladder (in both epithelial and smooth muscle layers) at much higher levels than in other human and mouse tissues. Hydrophobic bile salts can act as signaling molecules that bind to GPBAR1 on the PM of GBSM and lead to activation of the subsequent Gs-cAMP-PKA signaling pathway. This effect of hydrophobic bile salts will ultimately result in opening $K_{\text {ATP }}$ channels, cell membrane hyperpolarization and decreased GBSM contraction. $^{25,26}$

Recently, the discovery of interstitial Cajal-like cells (ICLCs) in the gallbladder provides new insight into the pathogenesis of impaired gallbladder motility. ${ }^{27-29}$ Gallbladder ICLCs are located almost exclusively within the muscularis propria, where they are electrically coupled with GBSM cells. ICLCs are believed to act as sources of spontaneous, electric slow waves responsible for paced contractions of GBSM, thus regulating the rhythm of gallbladder bile release. ${ }^{27,28,30}$ A significantly smaller number of ICLCs was found in gallbladders from patients with cholelithiasis compared with those without gallstones. ${ }^{31}$ Furthermore, a negative correlation between the number of ICLCs and bile CSI was discovered. ${ }^{32,33}$ It remains unknown whether lithogenic bile also demonstrates an adverse effect on ICLCs. There is evidence that a high cholesterol diet significantly reduces the expression of c-Kit, which is important in regulating the proliferation and survival of ICLCs. ${ }^{34}$ Other indirect inflammatory effects such as apoptotic mechanisms may also lead to the loss of ICLCs. The published research on gallbladder ICLCs remains limited. Elucidation of the role of ICLCs in the gallbladder may offer new avenues to prevent and treat gallbladder motility defects present in CGD.

\section{ION AND WATER ABSORPTION}

CSI has long been regarded as a reliable predictor for cholesterol nucleation. However, despite its higher CSI, hepatic bile from patients with cholesterol gallstones usually nucleates more slowly than associated (more concentrated) 
gallbladder bile. On the other hand, dilution of model bile leads to progressively longer nucleation time. These phenomena demonstrate that bile concentration is another important factor for nucleation of cholesterol monohydrate crystals. ${ }^{35}$

After secretion by the hepatocyte into the canalicular space, cholesterol is transported mainly in cholesterolphospholipid vesicles. As nascent hepatic bile is increasingly concentrated during bile transfer along the bile ducts to the gallbladder, cholesterol and phospholipids begin to transfer from vesicles to mixed micelles, which contain a mixture of cholesterol, phospholipids and bile salts. The preference for phospholipid transfer leads to a high cholesterol/phospholipid ratio and reduced stability of the residual vesicles. Therefore, cholesterol monohydrate is inclined to crystallize from these supersaturated vesicular fractions. ${ }^{36,37}$ The major function of the gallbladder is not only to store bile but also to concentrate it by absorption of water and ions. This function of the gallbladder, in conjunction with hepatic hypersecretion of cholesterol, contributes another prerequisite for cholesterol gallstone formation.

Hepatic bile is concentrated four to fivefold by the normal fasting gallbladder, thereby leading to exceedingly high concentrations of bile lipids (mainly cholesterol, bile salts, and phospholipids) in gallbladder bile. ${ }^{35}$ Abnormal gallbladder concentrating function is observed in experimental cholelithiasis at the point when cholesterol crystals are present but before the formation of gallstones. ${ }^{38}$ The fasting gallbladder to hepatic bile ratios for individual and total biliary lipids are a rough estimate of the capacity of gallbladder concentration and are uniformly increased in animals on a lithogenic diet compared with those on a control diet. ${ }^{39}$ The most likely explanation for these findings is increased gallbladder absorption of ions and water. Enhanced gallbladder absorption of water and ions has also been demonstrated in more direct studies using in vivo dual isotope technique. ${ }^{38}$ Moreover, enhanced water absorption by the gallbladder is observed under conditions such as pregnancy or truncal vagotomy, which are both associated with an increased incidence of gallstones in human subjects. ${ }^{40,41}$ Increase in gallbladder absorption may also promote gallstone formation by concentrating pro-nucleating factors and increasing bile viscosity. Reducing gallbladder concentrating function by administration of amiloride, an inhibitor of $\mathrm{Na}^{+}$absorption, could significantly decrease the incidence of cholesterol gallstones in animals fed with a lithogenic diet. ${ }^{42}$ It has long been debated whether such an abnormality is the result of enhanced gallbladder absorptive efficiency or merely the consequence of increased exposure time of bile to the mucosa secondary to gallbladder hypomotility. Regardless of the causative factors, the findings that dietary factors alter gallbladder absorption imply that gallstones are the result of impaired gallbladder mucosal and/or smooth muscle function.

It is easily understood that gallbladder hypomotility can lead to bile hyperconcentration. Some details in the regulation of gallbladder ion transport have been explored and provide further information regarding the cellular mechanisms behind the enhanced absorption efficiency of gallbladder epithelium. The removal of water from gallbladder bile involves different epithelial ion transport systems that lead to net $\mathrm{NaCl}$ apical absorption ${ }^{43-46}$ (Figure 1). Previous investigations in a variety of species demonstrate that gallbladder $\mathrm{Na}^{+}$and $\mathrm{Cl}^{-}$absorption is predominantly mediated by the parallel $\mathrm{Na}^{+} / \mathrm{H}^{+}$exchanger (NHE) and $\mathrm{Cl}^{-} / \mathrm{HCO}_{3}{ }^{-}$anion exchanger (AE) on the apical membrane of gallbladder epithelial cells (GBECs). These two exchangers are functionally correlated through intracellular $\mathrm{pH}$ and bicarbonate concentration. In healthy animals, protein kinase C, $\mathrm{Ca}^{2+}$-calmodulin, and protein kinase A hinder gallbladder concentrating function by inhibiting the rate of $\mathrm{Na}^{+}$and $\mathrm{Cl}^{-}$absorption. ${ }^{47-49}$ In response to a lithogenic diet, ion transport is released from these inhibitory factors, resulting in enhanced gallbladder absorption. ${ }^{38,50,51}$ A study by Giurgiu et $a l^{52}$ has demonstrated an increased net $\mathrm{Na}^{+}$absorption during the early pre-crystal stage of gallstone formation. The observed increase in $\mathrm{Na}^{+}$absorption was shown to be the result of an increase in gallbladder NHE activity..$^{53}$ Further studies on specific NHE isoforms demonstrated that both $\mathrm{NHE}_{2}$ and $\mathrm{NHE}_{3}$ expression increased during cholesterol gallstone formation. Furthermore, a shift in transport activity from $\mathrm{NHE}_{2}$ to $\mathrm{NHE}_{3}$ occurs simultaneously. However, the moderate increase in $\mathrm{NHE}_{2}$ and $\mathrm{NHE}_{3}$ expression at protein levels cannot fully account for the marked increase in total NHE activity. This suggests enhanced localization of preexisting NHE from the intracellular pool to the apical membrane. Recently, we have found that cholesterol gallstone

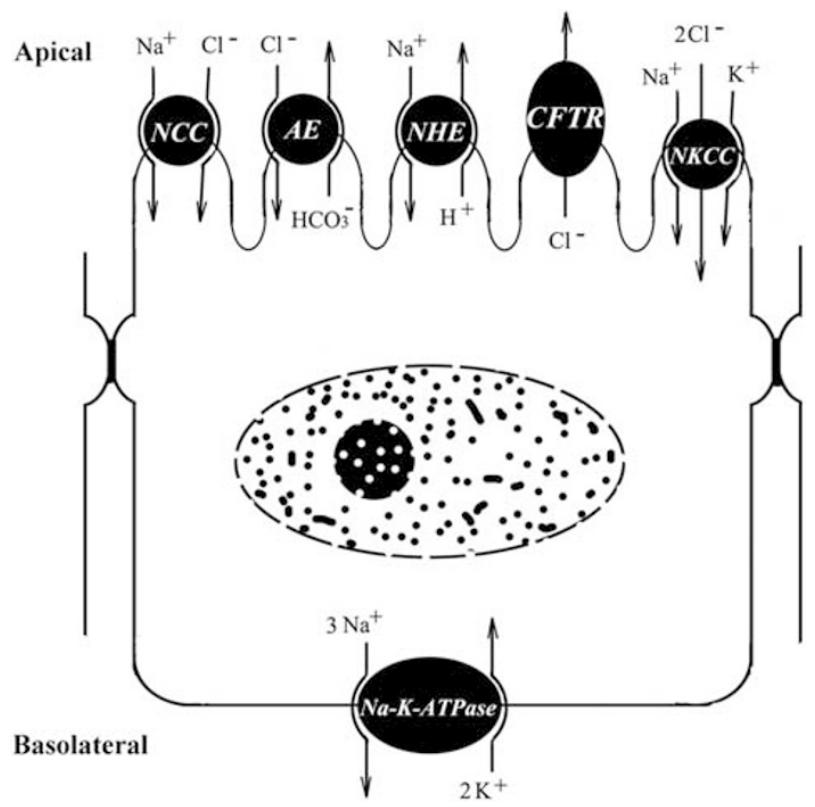

Figure 1 Working model of carriers and channels that sustain ion absorption and secretion in the gallbladder epithelium. 
formation was associated with a different expression of phosphorylated $\mathrm{NHE}_{3}$ at serine-552 $\left(\mathrm{P}-\mathrm{NHE}_{3}\right)$ in mouse GBECs (unpublished data). Given previous findings that $\mathrm{NHE}_{3}$ activity is related to its phosphorylation state, our results suggest alterations in NHE regulation during gallstone formation, probably via the exchanger trafficking mechanism. ${ }^{54,55}$ Furthermore, AE, which is usually co-expressed with NHE on absorptive epithelium, has a synergistic effect with NHE in $\mathrm{Na}^{+}$and $\mathrm{Cl}^{-}$absorption. Concomitant abnormality in AE activity is expected when NHE function is altered. On the other hand, the gallbladder also has secretive function that is mainly mediated by cystic fibrosis transmembrane conductance regulator (CFTR) ${ }^{56}$ Decrease in the secretive function of the epithelium quite possibly translates into enhanced absorption. Thus, the existence of other transporters in GBECs, especially CFTR, may also have a role in the pathogenesis of CGD.

The initiating factors that lead to the observed dysfunctional gallbladder ion transport are not clear. Since these changes occur only when cholesterol-supersaturated bile is present in the gallbladder, direct exposure of the gallbladder mucosa to lithogenic bile is highly suspected to be the trigger event. Ammon ${ }^{57}$ has shown that bile salts, at concentrations well below those usually present in the gallbladder, have a stimulating effect on $\mathrm{Cl}^{-}$secretion from GBECs, thus inhibiting net water absorption by gallbladder epithelium. Another study by Chignard et al $l^{58}$ that exposed cultured human GBECs to taurochenodeoxycholate and tauroursodeoxycholate demonstrated the prosecretory effects of these bile salts, both of which markedly accelerate the secretion of $\mathrm{Cl}^{-}$through potentiating the AC-cAMP signaling pathway. These effects can be reversed by the addition of phospholipids. These observations suggest that the increase in the ratio of the biliary concentration of phospholipids to bile salts, as happens in lithogenic bile, may be the trigger event of enhanced gallbladder absorption during early cholesterol gallstone formation. Moreover, exposure of the gallbladder epithelium to cholesterol-supersaturated bile may increase the cholesterol content of GBECs and affect PM fluidity. A link between $\mathrm{PM}$ cholesterol content and $\mathrm{NHE}_{3}$ activity has been suggested by numerous studies, which have shown that reducing cholesterol content of lipid raft with $\mathrm{M} \beta \mathrm{CD}$ may decrease cellular $\mathrm{Na}^{+} / \mathrm{H}^{+}$exchange by influencing $\mathrm{NHE}_{3}$ trafficking. ${ }^{59,60}$ This effect of $\mathrm{M} \beta \mathrm{CD}$ can be completely reversed by repletion of cholesterol. It is then entirely reasonable to suppose that high PM cholesterol content could result in increased $\mathrm{NHE}_{3}$ activity and enhanced gallbladder absorption. Alternatively, membrane cholesterol enrichment may alter ion transport activity by interfering with cell signal transduction. ${ }^{61-63}$ It remains unknown whether enhanced gallbladder absorption is due to aberrant signaling transduction in GBECs induced by abnormal bile salt concentrations or the result of altered PM fluidity largely due to increased cholesterol content. Additional investigations are required to identify specific constituents of lithogenic bile that induce the observed alterations in gallbladder absorption and the underlying mechanisms.

The recognition of the expression of aquaporins (AQP1 and AQP8) in GBECs provides us new insight into the osmotic movement of water through the epithelium. The presence of two distinct AQPs in the apical membrane of GBECs may give rise to the speculation that gallbladder epithelium could absorb and secrete fluid via separate AQPs. ${ }^{64}$ However, a study using a gene knockout technique showed no difference in either bile osmolality or bile salt concentration in gallbladders from wild-type and AQP1 knockout mice. These findings argue against an important role for AQP1 in mouse gallbladder. ${ }^{65}$ However, the synchronous decrease in AQP1 and AQP8 protein expression and gallbladder concentrating function after cholesterol gallstone formation in C57L mice indicates their relation to lithogenesis. ${ }^{66}$ The actual role in lithogenesis and potential therapeutic implications of gallbladder AQPs remain to be explored.

\section{LIPID TRANSPORT AND CSI}

The most recognized function of the gallbladder is to store and concentrate bile. However, this organ is not just a passive container; it can also actively modify bile composition by both absorption and secretion of lipids. Simultaneous influx and efflux of cholesterol occurs at the apical PM of GBECs via both physico-chemical and protein-mediated mechanisms. ${ }^{67}$ The net direction of movement of cholesterol depends more on the lipid composition of gallbladder bile and hydrophobicity of bile salts than on the mucosa, with supersaturated bile (CSI $>1)$ and more hydrophobic bile salts resulting in net absorption of cholesterol into the tissue. ${ }^{68}$ As bile is concentrated in the gallbladder, the increase in concentration of cholesterol is only $71 \%$ that of bile salts. Taking into consideration the fact that bile salts are only minimally absorbed by the gallbladder, these findings suggest a $29 \%$ net absorption of cholesterol. ${ }^{67}$ Using in vitro-isolated preparations of the human gallbladder, Corradini and co-workers ${ }^{69}$ also demonstrated that gallbladder epithelium absorbs $23 \%$ of cholesterol but only a net $9 \%$ of bile salts during a period of $5 \mathrm{~h}$. Consequently, the cholesterol molar percentage is significantly reduced, while the bile salts molar percentage is significantly increased. The lipid modulating ability of the gallbladder epithelium is thus regarded as a major determinant of gallbladder bile CSI. Biliary cholesterol supersaturation is regarded as the principal cause of CGD when there is excess cholesterol or not enough solubilizing bile salts and phospholipids. The gallbladder epithelium can significantly alter the composition of bile. This process in itself could act as a protective mechanism against gallstone formation.

Normal gallbladder epithelium may have a positive effect on gallbladder bile and hence an ability to prevent cholesterol supersaturation; this ability is impaired in patients with CGD, however. ${ }^{70}$ To better clarify the influence that gallbladder epithelium exerts on bile, a more direct methodological 
approach has been developed by Corradini et al ${ }^{71}$ using the in vitro-isolated, intra-arterially perfused gallbladder model. Their results demonstrated that cholesterol absorption by the gallbladder was markedly reduced in cholesterol gallstone patients compared with those in controls, whereas bile salt absorption was less affected. Consequently, gallstone gallbladders induced a more lithogenic bile with significant increase of cholesterol and decrease in bile salt molar percentages due to impaired lipid absorption. In combination with hepatic cholesterol hypersecretion, this defect in lipid absorption represents an additional contributing factor to cholesterol gallstone formation.

Significant advances have been made in understanding cholesterol uptake, intracellular trafficking, and efflux mechanisms of gallbladder epithelium. Thus far, ATP-binding cassette transporters G5/G8 (ABCG5/G8), ${ }^{72}$ megalin and cubilin, ${ }^{73}$ scavenger receptor class $\mathrm{B}$ type I (SR-BI), ${ }^{74}$ and $A B C A 1^{75}$ have been reported to be expressed in GBECs. The obligate heterodimers ABCG5/G8 are expressed on the apical membrane of epithelial cells, where they excrete cholesterol into the lumen. Megalin and cubilin are large, multipleligand receptors that can mediate cholesterol uptake from the apical surface of epithelial cells. SR-BI, mainly known as the selective uptake receptor for cholesterol, also has a role in apical cholesterol uptake. ABCA1 is expressed on the basolateral membrane of GBECs and initiates cholesterol efflux out of the epithelial cells (Figure 2). Changes in the activities of these proteins could conceivably influence cholesterol

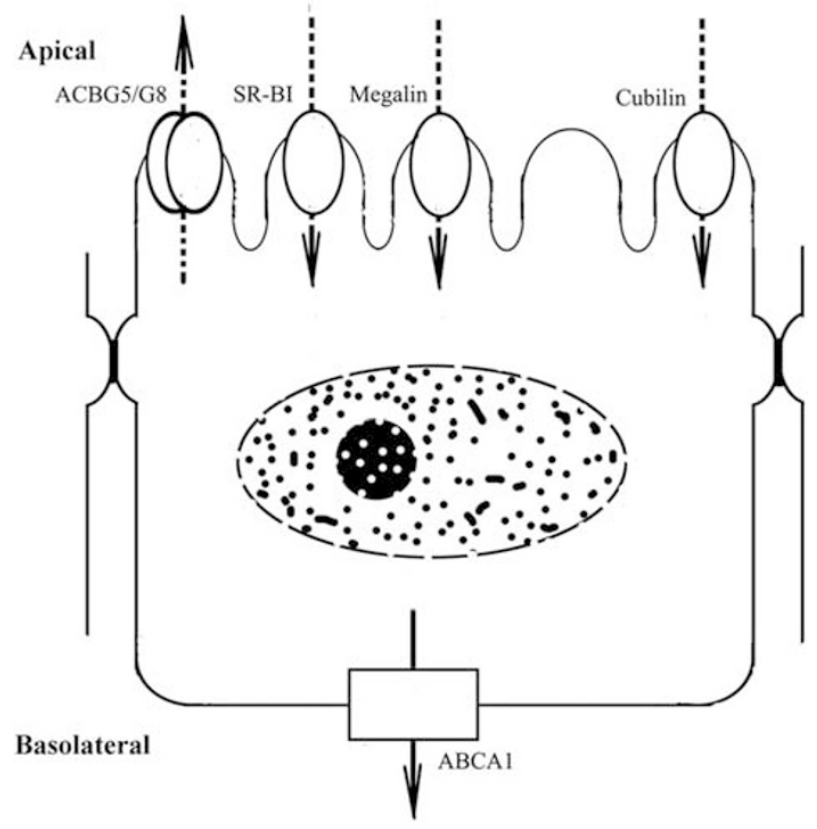

Figure 2 Cholesterol transporters in the gallbladder epithelial cells (GBECs). Transport proteins are visualized as symbols. Known transfer directions are shown in solid lines, while hypothetical transfer directions are shown in dotted lines. transport by GBECs. However, the functionality of the above transporters in GBECs has not yet been studied in animal experiments or human materials. Their transport directions are all derived from their function in liver and intestine. Studies on normal and diseased gallbladders have helped clinicians gain some insight into their functional role with respect to CGD, however, except for SR-BI, whose expression is restricted to the apical membrane of GBECs, ${ }^{76}$ intracellular signals were noted for $\mathrm{ABCG} 5 / \mathrm{G} 8,{ }^{77} \mathrm{ABCA} 1,{ }^{78}$ megalin, and cubilin $^{73}$ by immunohistochemistry studies. These findings suggest a trafficking mechanism in their transport activity regulation. Specially, the subcellular localization of ABCG5/ G8 in GBECs does show alterations, with predominantly intracellular localization at baseline and predominantly apical localization following short-term $(24 \mathrm{~h})$ cholesterol loading with model bile or LXRa/RXR ligand treatment. ${ }^{72}$ Furthermore, ABCA1, ABCG5/G8, and megalin, but not cubilin, are more highly expressed at protein levels in gallbladders with cholesterol gallstones than in normal gallbladders. ${ }^{73,78}$ In contrast, increasing the bile cholesterol content with a lithogenic diet leads to a marked decrease in expression of SR-BI in the murine gallbladder. ${ }^{76}$

The expression of the above transporters is in agreement with a previous study that both cholesterol absorption and secretion occur on the apical side of GBECs. ${ }^{68}$ In addition, their existence confirms the important role of the gallbladder in regulating biliary cholesterol content. CGD is usually associated with increased expression of cholesterol efflux transporters (ABCA1 and ABCG5/G8) and decreased expression of cholesterol influx transporters (SR-BI). There is good reason to suspect that altered expression in these transporters is responsible for impaired gallbladder cholesterol transport, which may facilitate cholesterol precipitation by increasing CSI. ${ }^{78,79}$ However, we cannot rule out the possibility that the differential expression of cholesterol transporters in the diseased gallbladder may merely reflect cellular protective mechanisms against excessive cellular cholesterol absorption. In either case, the distribution pattern of these cholesterol transporters suggests a mainly apical-to-basolateral cholesterol transport direction by GBECs to prevent cholesterol overload and gallstone formation. ${ }^{80}$ Subsequent in vitro functional assays for each of these proteins would provide a clearer view into gallbladder cholesterol transport and their relation to cholesterol gallstone formation.

\section{CHOLESTEROL NUCLEATION PROMOTING AND INHIBITING PROTEINS}

Of all the tissues in the body, the gallbladder epithelium is uniquely positioned to face the highest concentrations of bile lipids for the longest time. To cope with this situation, GBECs secrete special proteins, either to protect themselves against the detergent effect of a high concentration of bile salts or to prevent cellular cholesterol overload by facilitating its apical-to-basolateral efflux..$^{81,82}$ Nevertheless, this function becomes impaired when GBECs are challenged by lithogenic 
bile, which leads to alterations in the secretion of these proteins and an imbalance between cholesterol nucleation promoting and inhibiting factors.

Hypersecretion of pro-nucleating mucins (MUC) by GBECs has long been suggested as a contributing factor for gallstone formation. ${ }^{83-85}$ Multiple MUC genes are expressed in GBECs, with their products including gel-forming MUCs (MUC2, MUC5AC, MUC5B, and MUC6) and membranebound MUCs (MUC1, MUC3A, MUC3B, MUC4, and MUPCDH) ${ }^{86}$ Gel-forming MUCs may provide a favorable environment for gallstone formation by accelerating the nucleation of cholesterol monohydrate crystals from supersaturated bile. In addition, there is evidence that membranebound MUC (MUC1) can also promote gallstone formation by augmenting gallbladder cholesterol absorption and impairing gallbladder motility. ${ }^{87,88}$ Increase in gallbladder MUC secretion has been demonstrated in many animal models as well as in patients with CGD..$^{89,90}$ Once again, this phenomenon occurs in the earliest stages of CGD before the appearance of cholesterol crystals. Decreasing MUC secretion with aspirin prevents gallstone formation in animal models and decreases the risk of gallstone recurrence following dissolution therapy in humans. ${ }^{91,92} \mathrm{New}$ progress has been made to identify the initiating event that triggers the observed MUC hypersecretion in recent years. A shift in the bile salt composition toward more hydrophobic, as occurs in lithogenic bile, is more effective at stimulating MUC secretion. This effect of hydrophobic bile salts seems to occur independently of their detergent properties. In contrast, the more hydrophilic salt ursodeoxycholic acid has an inhibitory effect on MUC secretion. ${ }^{93}$ In addition, other factors such as inflammatory cytokines, bacterial products, and growth factors can also lead to mucin hypersecretion. ${ }^{94-97}$

Biliary apolipoprotein A-I (apoA-I), which can be synthesized by GBECs, possesses nucleation inhibiting properties. ${ }^{98}$ At concentrations present in bile, apoA-I can directly retard cholesterol crystal nucleation when added to model systems of supersaturated bile. ${ }^{99}$ In addition, apoA-I can also elicit basolateral cholesterol efflux via the ABCA1-mediated pathway and enhance its removal from gallbladder bile. ${ }^{75}$ However, bile samples from gallstone patients exhibit reduced Apo A-I concentrations when compared with those from gallstone-free patients. ${ }^{100}$

\section{SUMMARY}

The basic role of the gallbladder is to enhance the digestive power of bile through a concentrating mechanism, drive the flow of bile salt through the enterohepatic circulation via periodic motion, and maintain the thermodynamic stability of gallbladder bile through differential absorption of lipids. These functions of the gallbladder are all impaired and constitute essential contributing factors in CGD. While recognizing that the pathogenesis of CGD is multifactorial, the mechanisms behind the defects in gallbladder function have not yet been fully illuminated. Attempts to describe these aspects of gallbladder function may improve our understanding of the natural history of CGD and enable the development of new treatment strategies.

\section{ACKNOWLEDGMENTS}

This work was not supported by any outside research funding.

\section{DISCLOSURE/CONFLICT OF INTEREST}

The authors declare no conflict of interest.

1. LaMorte WW, Schoetz Jr DJ, Birkett $\mathrm{DH}$, et al. The role of the gallbladder in the pathogenesis of cholesterol gallstones. Gastroenterology 1979;77:580-592.

2. Small DM, Rapo S. Source of abnormal bile in patients with cholesterol gallstones. N Engl J Med 1970;283:53-57.

3. Dikkers A, Tietge UJ. The neglected cousin of the hepatocyte: how gallbladder epithelial cells might contribute to cholesterol gallstone formation. Dig Dis Sci 2013;58:296-298.

4. van Erpecum $\mathrm{KJ}$, Wang DQ. The gallbladder: innocent bystander or major factor in cholesterol-gallstone formation? J Lab Clin Med 2005; 146:202-204.

5. Fridhandler TM, Davison JS, Shaffer EA. Defective gallbladder contractility in the ground squirrel and prairie dog during the early stages of cholesterol gallstone formation. Gastroenterology 1983;85: 830-836.

6. Kishk SM, Darweesh RM, Dodds WJ, et al. Sonographic evaluation of resting gallbladder volume and postprandial emptying in patients with gallstones. AJR Am J Roentgenol 1987;148:875-879.

7. Li YF, Moody FG, Weisbrodt NW, et al. Gallbladder contractility and mucus secretion after cholesterol feeding in the prairie dog. Surgery 1986;100:900-904.

8. Lavoie B, Nausch B, Zane EA, et al. Disruption of gallbladder smooth muscle function is an early feature in the development of cholesterol gallstone disease. Neurogastroenterol Motil 2012;24:e313-e324.

9. Xu QW, Shaffer EA. Cisapride improves gallbladder contractility and bile lipid composition in an animal model of gallstone disease. Gastroenterology 1993;105:1184-1191.

10. Xu QW, Scott RB, Tan DT, et al. Effect of the prokinetic agent, erythromycin, in the Richardson ground squirrel model of cholesterol gallstone disease. Hepatology 1998;28:613-619.

11. Xu QW, Mantle M, Pauletzki JG, et al. Sustained gallbladder stasis promotes cholesterol gallstone formation in the ground squirrel. Hepatology 1997;26:831-836.

12. Pauletzki J, Althaus R, Holl J, et al. Gallbladder emptying and gallstone formation: a prospective study on gallstone recurrence. Gastroenterology 1996;111:765-771.

13. Behar J, Lee KY, Thompson WR, et al. Gallbladder contraction in patients with pigment and cholesterol stones. Gastroenterology 1989:97:1479-1484

14. Chen $Q$, Chitinavis V, Xiao $Z$, et al. Impaired $G$ protein function in gallbladder muscle from progesterone-treated guinea pigs. Am J Physiol 1998;274:G283-G289.

15. Xiao Z, Chen Q, Amaral J, et al. CCK receptor dysfunction in muscle membranes from human gallbladders with cholesterol stones. Am J Physiol 1999;276:G1401-G1407.

16. Yu $\mathrm{P}$, Chen $\mathrm{Q}$, Harnett $\mathrm{KM}$, et al. Direct $\mathrm{G}$ protein activation reverses impaired CCK signaling in human gallbladders with cholesterol stones. Am J Physiol 1995;269:G659-G665.

17. Chen $Q$, Amaral J, Biancani $P$, et al. Excess membrane cholesterol alters human gallbladder muscle contractility and membrane fluidity. Gastroenterology 1999;116:678-685.

18. Mansour A, Dawoud I, Gad-El-Hak N. The potential site of disordered gallbladder contractility during the early stage of cholesterol gallstone formation. Hepatogastroenterology 1998;45:1404-1409.

19. Jennings LJ, Xu QW, Firth TA, et al. Cholesterol inhibits spontaneous action potentials and calcium currents in guinea pig gallbladder smooth muscle. Am J Physiol 1999;277:G1017-G1026.

20. Cong P, Pricolo V, Biancani $P$, et al. Effects of cholesterol on CCK-1 receptors and caveolin-3 proteins recycling in human gallbladder muscle. Am J Physiol Gastrointest Liver Physiol 2010;299:G742-G750. 
21. Xiao Z, Schmitz F, Pricolo VE, et al. Role of caveolae in the pathogenesis of cholesterol-induced gallbladder muscle hypomotility. Am J Physiol Gastrointest Liver Physiol 2007;292:G1641-G1649.

22. Xiao ZL, Amaral J, Biancani P, et al. Impaired cytoprotective function of muscle in human gallbladders with cholesterol stones. Am J Physiol Gastrointest Liver Physiol 2005;288:G525-G532.

23. Xiao ZL, Biancani P, Behar J. Role of PGE2 on gallbladder muscle cytoprotection of guinea pigs. Am J Physiol Gastrointest Liver Physiol 2004;286:G82-G88.

24. Xiao ZL, Rho AK, Biancani $P$, et al. Effects of bile acids on the muscle functions of guinea pig gallbladder. Am J Physiol Gastrointest Liver Physiol 2002;283:G87-G94.

25. Lavoie B, Balemba OB, Godfrey $C$, et al. Hydrophobic bile salts inhibit gallbladder smooth muscle function via stimulation of GPBAR1 receptors and activation of KATP channels. J Physiol 2010;588:3295-3305.

26. Li T, Holmstrom SR, Kir S, et al. The $G$ protein-coupled bile acid receptor, TGR5, stimulates gallbladder filling. Mol Endocrinol 2011;25: 1066-1071.

27. Huang Y, Mei F, Yu B, et al. Distribution of the interstitial Cajal-like cells in the gallbladder and extrahepatic biliary duct of the guinea-pig. Acta Histochem 2009;111:157-165.

28. Pasternak A, Gajda M, Gil K, et al. Evidence of inter stitial Cajal-like cells in human gallbladder. Folia Histochem Cytobiol 2012;50:581-585.

29. Ahmadi O, Nicholson Mde L, Gould ML, et al. Interstitial cells of Cajal are present in human extrahepatic bile ducts. J Gastroenterol Hepatol 2010;25:277-285.

30. $\mathrm{Xu} \mathrm{D}, \mathrm{Yu} B \mathrm{P}, \mathrm{Luo} \mathrm{HS}$, et al. Control of gallbladder contractions by cholecystokinin through cholecystokinin-A receptors on gallbladder interstitial cells of Cajal. World J Gastroenterol 2008;14:2882-2887.

31. Pasternak A, Gil K, Matyja A, et al. Loss of gallbladder interstitial Cajallike cells in patients with cholelithiasis. Neurogastroenterol Motil 2013;25:e17-e24.

32. Pasternak A, Matyja A, Gil K, et al. Interstitial cajal-like cells and bile lithogenicity in the pathogenesis of gall-stone disease. Pol Przegl Chir 2013;85:311-316

33. Matyja A, Gil K, Pasternak A, et al. Telocytes: new insight into the pathogenesis of gallstone disease. J Cell Mol Med 2013;17: 734-742.

34. Hu WM, Luo HS, Ding XW, et al. Expression of C-kit messenger ribonucleic acid and C-kit protein in the gallbladders in guinea pigs of high cholesterol diet. Dig Dis Sci 2009;54:1651-1655.

35. van Erpecum KJ, van Berge Henegouwen GP, Stoelwinder B, et al. Bile concentration is a key factor for nucleation of cholesterol crystals and cholesterol saturation index in gallbladder bile of gallstone patients. Hepatology 1990;11:1-6.

36. van Erpecum KJ. Biliary lipids, water and cholesterol gallstones. Biol Cell 2005;97:815-822.

37. Venneman NG, van Erpecum KJ. Pathogenesis of gallstones. Gastroenterol Clin North Am 2010;39:171-183.

38. Conter RL, Roslyn JJ, Porter-Fink V, et al. Gallbladder absorption increases during early cholesterol gallstone formation. Am J Surg 1986;151:184-191.

39. Roslyn JJ, Conter RL, DenBesten L. Altered gallbladder concentration of biliary lipids during early cholesterol gallstone formation. Dig Dis Sc 1987;32:609-614.

40. Rådberg G, Svanvik J. Influence of pregnancy, oophorectomy and contraceptive steroids on gall bladder concentrating function and hepatic bile flow in the cat. Gut 1986;27:10-14.

41. Björck S, Jansson R, Svanvik J. The concentrating function of the feline gallbladder after truncal vagotomy. Acta Chir Scand 1984;150: 393-397.

42. Strichartz SD, Abedin MZ, Abdou MS, et al. The effects of amiloride on biliary calcium and cholesterol gallstone formation. Ann Surg 1989;209:152-156.

43. Abedin $\mathrm{MZ}$, Giurgiu DI, Abedin $\mathrm{ZR}$, et al. Characterization of $\mathrm{Na}+/ \mathrm{H}+$ exchanger isoform (NHE1, NHE2, and NHE3) expression in prairie dog gallbladder. J Membr Biol 2001;182:123-134.

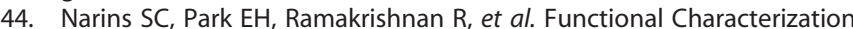
of $\mathrm{Na}^{+} / \mathrm{H}^{+}$Exchangers in Primary Cultures of Prairie Dog Gallbladder. Membr Biol 2004;197:123-134.

45. Cremaschi D, Porta C, Bottà G, et al. Apical $\mathrm{Na}^{+}-\mathrm{Cl}^{-}$symport in rabbit gallbladder epithelium: a thiazide-sensitive cotransporter (TSC). J Membr Biol 2000;176:53-65.
46. Meyer G, Guizzardi $F$, Rodighiero $S$, et al. Ion transport across the gallbladder epithelium. Curr Drug Targets Immune Endocr Metabol Disord 2005;5:143-151.

47. Cates JA, Abedin MZ, Saunders-Kirkwood KD, et al. Protein kinase C regulates prairie dog gallbladder ion transport. Surgery 1995;117: 206-212.

48. Moser AJ, Abedin MZ, Abedin ZR, et al. $\mathrm{Ca}^{2+}$ calmodulin regulates basal gallbladder absorption. Surgery 1993;114:300-307.

49. Petersen KU, Wehner F, Winterharger JM. Na/H exchange at the apical membrane of guinea pig gallbladder epithelium:properties and inhibition by CAMP. Pflugers Arch 1985;405(Suppl 1):S115-S120.

50. Moser AJ, Giurgiu DI, Morgenstern KE, et al. Calmodulin regulation of gallbladder ion transport becomes dysfunctional during gallstone formation in prairie dogs. Dig Dis Sci 2000;45:1422-1430.

51. Narins SC, Ramakrishnan R, Park EH, et al. Protein kinase $C$ regulation of gallbladder $\mathrm{Na}+$ transport becomes progressively dysfunctional during gallstone formation. J Lab Clin Med 2005;146:227-237.

52. Giurgiu DI, Saunders-Kirkwood KD, Roslyn JJ, et al. Sequential changes in biliary lipids and gallbladder ion transport during gallstone formation. Ann Surg 1997;225:382-390.

53. Narins SC, Ramakrishnan $\mathrm{R}$, Park $\mathrm{EH}$, et al. Gallbladder $\mathrm{Na}^{+} / \mathrm{H}^{+}$ exchange activity is up-regulated prior to cholesterol crystal formation. Eur J Clin Invest 2005;35:514-522.

54. Crajoinas RO, Lessa LM, Carraro-Lacroix LR, et al. Posttranslational mechanisms associated with reduced NHE3 activity in adult vs. young prehypertensive SHR. Am J Physiol Renal Physiol 2010;299: F872-F881.

55. Yang LE, Sandberg MB, Can AD, et al. Effects of dietary salt on renal $\mathrm{Na}^{+}$transporter subcellular distribution, abundance, and phosphorylation status. Am J Physiol Renal Physiol 2008;295:F1003-F1016.

56. Bazzini C, Bottà G, Meyer G, et al. The presence of NHE1 and NHE3 $\mathrm{Na}^{+}-\mathrm{H}^{+}$exchangers and an apical cAMP-independent $\mathrm{Cl}^{-}$channel indicate that both absorptive and secretory functions are present in calf gall bladder epithelium. Exp Physiol 2001;86:571-583.

57. Ammon HV. Effect of taurine conjugated bile salts with and without lecithin on water and electrolyte transport in the canine gallbladder in vivo. Gastroenterology 1979;76:778-783.

58. Chignard N, Mergey M, Veissière $D$, et al. Bile salts potentiate adenylyl cyclase activity and CAMP-regulated secretion in human gallbladder epithelium. Am J Physiol Gastrointest Liver Physiol 2003;284: G205-G212.

59. Li X, Galli T, Leu S, et al. $\mathrm{Na}^{+}-\mathrm{H}^{+}$exchanger 3 (NHE3) is present in lipid rafts in the rabbit ileal brush border: a role for rafts in trafficking and rapid stimulation of NHE3. J Physiol 2001;537:537-552.

60. Murtazina $\mathrm{R}$, Kovbasnjuk $\mathrm{O}$, Donowitz $\mathrm{M}$, et al. $\mathrm{Na}^{+} / \mathrm{H}^{+}$exchanger NHE3 activity and trafficking are lipid Raft-dependent. J Biol Chem 2006;281:17845-17855.

61. Shi Q, Hou Y, Hou J, et al. Glycyrrhetic acid synergistically enhances $\beta_{2}$ adrenergic receptor-Gs signaling by changing the location of $\mathrm{G} \alpha \mathrm{s}$ in lipid rafts. PLoS One 2012;7:e44921.

62. Zhou MH, Yang G, Jiao $\mathrm{S}$, et al. Cholesterol enhances neuron susceptibility to apoptotic stimuli via CAMP/PKA/CREB-dependent up-regulation of Kv2.1. J Neurochem 2012;120:502-514.

63. Peres C, Yart A, Perret B, et al. Modulation of phosphoinositide 3-kinase activation by cholesterol level suggests a novel positive role for lipid rafts in lysophosphatidic acid signalling. FEBS Lett 2003;534: 164-168.

64. Calamita G, Ferri D, Bazzini C, et al. Expression and subcellular localization of the AQP8 and AQP1 water channels in the mouse gallbladder epithelium. Biol Cell 2005;97:415-423.

65. Li L, Zhang H, Ma T, et al. Very high aquaporin-1 facilitated water permeability in mouse gallbladder. Am J Physiol Gastrointest Liver Physiol 2009;296:G816-G822.

66. van Erpecum KJ, Wang DQ, Moschetta A, et al. Gallbladder histopathology during murine gallstone formation: relation to motility and concentrating function. J Lipid Res 2006;47:32-41.

67. Shiffman ML, Sugerman HJ, Moore EW. Human gallbladder mucosal function. Effect of concentration and acidification of bile on cholesterol and calcium solubility. Gastroenterology 1990;99: 1452-1459.

68. Hayashi A, Lee SP. Bidirectional transport of cholesterol between gallbladder epithelial cells and model bile. Am J Physiol 1996;271: G410-G414. 
69. Ginanni Corradini S, Ripani C, Della Guardia P, et al. The human gallbladder increases cholesterol solubility in bile by differential lipid absorption: a study using a new in vitro model of isolated intraarterially perfused gallbladder. Hepatology 1998;28:314-322.

70. Ginanni Corradini S, Yamashita G, Nuutinen $\mathrm{H}$, et al. Human gallbladder mucosal function: effects on intraluminal fluid and lipid composition in health and disease. Dig Dis Sci 1998;43:335-343.

71. Corradini SG, Elisei W, Giovannelli L, et al. Impaired human gallbladder lipid absorption in cholesterol gallstone disease and its effect on cholesterol solubility in bile. Gastroenterology 2000;118:912-920.

72. Tauscher A, Kuver R. ABCG5 and ABCG8 are expressed in gallbladder epithelial cells. Biochem Biophys Res Commun 2003;307: 1021-1028.

73. Erranz B, Miquel JF, Argraves WS, et al. Megalin and cubilin expression in gallbladder epithelium and regulation by bile acids. J Lipid Res 2004:45:2185-2198.

74. Johnson MS, Svensson PA, Borén J, et al. Expression of scavenger receptor class B type I in gallbladder columnar epithelium. J Gastroenterol Hepatol 2002;17:713-720.

75. Lee J, Shirk A, Oram JF, et al. Polarized cholesterol and phospholipid efflux in cultured gall-bladder epithelial cells: evidence for an ABCA1mediated pathway. Biochem J 2002;364:475-484.

76. Miquel JF, Moreno M, Amigo L, et al. Expression and regulation of scavenger receptor class B type I (SR-BI) in gall bladder epithelium. Gut 2003:52:1017-1024.

77. Klett EL, Lee $M H$, Adams DB, et al. Localization of ABCG5 and ABCG8 proteins in human liver, gallbladder and intestine. BMC Gastroenterol 2004:4:21

78. Yoon JH, Choi HS, Jun DW, et al. ATP-binding cassette sterol transporters are differentially expressed in normal and diseased human gallbladder. Dig Dis Sci 2013:58:431-439.

79. Tsaroucha AK, Chatzaki E, Lambropoulou M, et al. Megalin and cubilin in the human gallbladder epithelium. Clin Exp Med 2008;8:165-170.

80. Lee J, Hong EM, Byun HW, et al. The effect of PPARa and PPARc ligands on inflammation and $A B C A 1$ expression in cultured gallbladder epithelial cells. Dig Dis Sci 2008;53:1707-1715.

81. Chuang SC, Hsi E, Lee KT. Mucin genes in gallstone disease. Clin Chim Acta 2012;413:1466-1471.

82. Secknus R, Darby GH, Chernosky A, et al. Apolipoprotein A-I in bile inhibits cholesterol crystallization and modifies transcellular lipid transfer through cultured human gall-bladder epithelial cells. J Gastroenterol Hepatol 1999;14:446-456.

83. Rege RV, Prystowsky JB. Inflammation and a thickened mucus layer in mice with cholesterol gallstones. J Surg Res 1998;74:81-85.

84. Kim HJ, Kim SH, Chae GB, et al. Increased expression of mucin $5 A C$ mRNA and decreased expression of epidermal growth-factor receptor mRNA in gallstone patients. Tohoku J Exp Med 2008;214:139-144.
85. Wilhelmi M, Jüngst $C$, Mock $M$, et al. Effect of gallbladder mucin on the crystallization of cholesterol in bile. Eur J Gastroenterol Hepatol 2004:16:1301-1307.

86. Bar Dayan Y, Vilkin A, Niv Y. Gallbladder mucin plays a role in gallstone formation. Eur J Intern Med 2004;15:411-414.

87. Wang $\mathrm{HH}$, Afdhal NH, Gendler SJ, et al. Targeted disruption of the murine mucin gene 1 decreases susceptibility to cholesterol gallstone formation. J Lipid Res 2004;45:438-447.

88. Wang $\mathrm{HH}$, Afdhal $\mathrm{NH}$, Gendler $\mathrm{SJ}$, et al. Evidence that gallbladder epithelial mucin enhances cholesterol cholelithogenesis in MUC1 transgenic mice. Gastroenterology 2006;131:210-222.

89. Lee SP, Lamont JT, Carey MC. Role of gallbladder mucin hypersecretion in the evolution of cholesterol gallstones. J Clin Invest 1981;67:1712-1723.

90. Messing B, Boires C, Kuntstlinger F, et al. Does total parentral nutrition induce gallbladder sludge formation and lithiasis? Gastroenterology 1983;84:1012-1019.

91. Lee SP, Carey MC, LaMont JT. Aspirin prevention of cholesterol gallstone formation in prairie dogs. Science 1981;211:1429-1431.

92. Hood K, Gleeson D, Ruppin DC, et al. Prevention of gallstone recurrence by non-steroidal anti-inflammatory drugs. Lancet 1988;2:1223-1225.

93. Jüngst C, Sreejayan N, Zündt B, et al. Ursodeoxycholic acid reduces lipid peroxidation and mucin secretagogue activity in gallbladder bile of patients with cholesterol gallstones. Eur J Clin Invest 2008;38:634-639.

94. Vilkin A, Nudelman I, Morgenstern S, et al. Gallbladder inflammation is associated with increase in mucin expression and pigmented stone formation. Dig Dis Sci 2007;52:1613-1620.

95. Maurer KJ, Carey MC, Fox JG. Roles of infection, inflammation, and the immune system in cholesterol gallstone formation. Gastroenterology 2009; 136:425-440

96. Zen Y, Harada K, Sasaki $\mathrm{M}$, et al. Lipopolysaccharide induces overexpression of MUC2 and MUC5AC in cultured biliary epithelial cells: possible key phenomenon of hepatolithiasis. Am J Pathol 2002;161:1475-1484.

97. Finzi L, Barbu V, Burgel PR, et al. MUC5AC, a gel-forming mucin accumulating in gallstone disease, is overproduced via an epidermal growth factor receptor pathway in the human gallbladder. Am J Pathol 2006;169:2031-2041.

98. Lee J, Tauscher A, Seo DW, et al. Cultured gallbladder epithelial cells synthesize apolipoproteins A-I and E. Am J Physiol Gastrointest Liver Physiol 2003;285:G630-G641.

99. Kibe A, Holzbach RT, LaRusso NF, et al. Inhibition of cholesterol crystal formation by apolipoproteins in supersaturated model bile. Science 1984:225:514-516.

100. Hasegawa T, Makino I. Measurement of apolipoprotein A1 in cholesterol gallstones and gallbladder bile of patients with gallstones. J Gastroenterol 1995;30:96-102. 